\title{
Imagery and sentence mediators in verbal discrimination learning*
}

\author{
EDWARD J. ROWE $\dagger$ and LESLIE J. CAKE \\ Memorial University of Newfoundland, St. John's, Newfoundland, Canada
}

\begin{abstract}
The effect of instructions to use imagery and sentence strategies in verbal discrimination learning (VDL) was investigated in two experiments. In Experiment $I$, both imaging to and constructing a sentence for the correct item of each pair facilitated VDL relative to an uninstructed control condition. Incorporating both words of the pair into a compound image or compound sentence had no effect, a result that was replicated in Experiment II. Of the two compound instructions, only compound sentences consistently facilitated associative recall of the VDL pairs, suggesting that compound imagery instructions were not always adhered to by the Ss.
\end{abstract}

Rowe and Paivio (1971a) have provided evidence for the facilitative effect of imagery instructions in verbal discrimination learning (VDL). In that experiment, different groups of Ss learned a list of high-imagery (High-I) words under instructions to either (a) image to only the right $(R)$ word of each pair or (b) form a compound image incorporating both the $\mathrm{R}$ and wrong (W) words, but at the same time "tagging" the image for the $\mathrm{R}$ word as the larger of the two. Performance in the single image condition surpassed that of the compound image group, as well as an uninstructed control condition and a repetition condition where the Ss overtly rehearsed the $\mathrm{R}$ and $\mathrm{W}$ items of each pair in a ratio of $4: 1$. The compound image condition did not differ from the control in VDL, but was the only condition that enhanced performance on a subsequent associative recall test.

A reliable effect of single image instructions on VDL has been verified in subsequent research (Levin, Ghatala, Wilder, \& Inzer, 1973; Rowe, 1972), but the situation with respect to compound imagery remains somewhat unclear. As pointed out by Rowe and Paivio (1971a), the finding that one type of compound image/ tagging strategy is ineffective does not disallow the possibility that some other form of compound imaging might be facilitative. The determination of whether or not this type of strategy can affect VDL is important in view of the striking effects that result from compound imagery in other memory tasks, especially paired associate learning (Paivio, 1971, Chapter 10). If compound imagery can be shown to produce different effects in VDL and paired associate learning (PAL), as the initial results suggest, this could help to theoretically extricate the processes involved in discrimination and associative learning of pairs. One purpose of the present study was to provide additional evidence on compound imagery effects in VDL through the use of instructions that were nonspecific with regard to ways in which the image corresponding to the $\mathrm{R}$ word could be tagged. A

\footnotetext{
*This research was supported by Grant A8580 from the National Research Council of Canada.

$\doteqdot$ Requests for reprints should be sent to Ldward J. Rowe. Department of Psychology. Memorial Iniversity of Newfoundland, St. John's, Newfoundland. Canada.
}

single image condition was also included in Experiment $\mathrm{I}$.

A second aim of the research was to explore the possible effects of two types of sentence mediators in VDL. These conditions were intended to parallel the two imagery instructions. Thus, the single sentence instructions encouraged the Ss to construct a sentence incorporating only the $\mathrm{R}$ word of each pair, and the compound sentence instructions emphasized producing a sentence to include both intrapair items. Mediators of the latter type are known to facilitate PAL (Paivio, 1971, Chapter 9) but, to the writers' knowledge, their effectiveness in VDL has not been assessed. Single sentence instructions also have apparently not been investigated, although the somewhat analogous procedure of requiring Ss to associate a real word with each $\mathrm{R}$ item in a list of CVC pairs enhances performance (Wike \& Wike, 1970).

The design of Experiment I included both High-I and low-imagery (Low-I) word pairs in addition to the two types of imagery and sentence instructions. Previous research in PAL (Paivio \& Foth, 1970) has demonstrated that imagery vs sentence mediation instructions have differential effects on High-I and Low-I pairs, with imagery being more effective in learning High-I items and sentences with Low-I. This interaction, which has a theoretical basis in Paivio's (1971) two-process theory of memory, might also obtain in VDL and/or associative recall. This is not a strong prediction, however, considering that special circumstances that were not present in Experiment I have been found necessary for the interaction in PAL (Paivio, 1971, Chapter 11).

\section{EXPERIMENT I}

Method

\section{Subjects}

The Ss were 60 undergraduates (30 males) at Memorial University. who were paid $\$ 1.50$ for participating in the experiment. All Ss were unfamiliar with the VDL task. They were assigned in rotation to one of the five instructional conditions: single imagery (SI). compound imagery (CI). single sentence (SS). compound sentence (CS). and an uninstructed control. with the restriction that there be an equal number of males and females in each group. 
Table 1

Mean Number of Errors in Verbal Discrimination Learning for Single Image (SI), Compound Image (CI), Single Sentence (SS). Compound Sentence (CS), and Control Conditions (Experiment I)

\begin{tabular}{cccccc}
\hline & \multicolumn{5}{c}{ Instruction } \\
\cline { 2 - 6 } Imagery & SI & CI & SS & CS & Control \\
\hline High-I & 0.83 & 5.17 & 0.50 & 3.33 & 6.92 \\
Low-I & 3.17 & 8.25 & 2.58 & 7.67 & 9.83 \\
Total & $4.00^{\mathrm{a}}$ & $13.42^{\mathrm{b}}$ & $3.08^{\mathrm{a}}$ & $11.00^{\mathrm{b}}$ & $16.75^{\mathrm{b}}$ \\
\hline
\end{tabular}

Vote- $a$ and $b$ indicate means did not differ significantly from others with the same superscript. Stondard deviations ranged from .76 to 4.31 .

\section{Lists}

Sixty-four two- and three-syllable words. four to eight letters in length, were selected from the Paivio. Yuille, and Madigan (1968) norms. The High-I words had I values greater than 6.06 and the Low-I had I values below 3.84. All of the words exceeded 32 per million by the Thorndike-Lorge frequency count. A mixed list of 16 High-I and 16 Low-I pairs was constructed by a procedure that minimized obvious intrapair associations but was otherwise nonsystematic.

\section{Procedure}

The Ss received two study-test trials on the VDL list. followed by an associative recall test and a questionnaire on the use of learning strategies during VDL. In the VDL phase of the experiment, the word pairs, typed on $3 \times 5 \mathrm{in}$. white index cards. were manually exposed by the $S$ for $6 \mathrm{sec}$ each. timed by a metronome. On the study trials. the $\mathrm{R}$ item of each pair was underlined, and $S$ was required to read both words aloud from left to right. In addition. Ss in the SI condition were instructed to form an image for each $\mathrm{R}$ word. The CI Ss were told to form an image for both words. making the image for the $R$ word in some way different from the image for the $W$ word. The SS group was instructed to silently construct a sentence that incorporated only the $\mathrm{R}$ word of each pair, while the CS group was told to construct a sentence incorporating both words. The control Ss were told simply to note to themselves which item of a pair was correct. All Ss were reminded of the mnemonic instructions prior to the second study trial. On the test trials, the pairs were presented without the underlining and the $S$ was required to name the $R$ word. Guessing was encouraged. Practice in the use of the various strategies was provided by two study-test trials on a separate list of four High-I and four Low-I pairs.

Four different random orders of the 32 pairs were used in presenting the experimental lists. The spatial position of $\mathbf{R}$ and II items was counterbalanced across orders such that, for any given study or test trial subsequent to the initial presentation. item position for half of the pairs was changed from the preceding trial but with the items of a given pair changing position an equal number of times (four) across all four orders. The $R$ and W items occurred equally of ten on the left and right for each presentation of the list. Half of the $S s$ in each cordition had one member of a pair correct, while the other half had the opposite item correct.

For the associative recall test. each $\mathrm{S}$ was given a response sheet with one word of each pair listed down the left-hand side of the page and a blank opposite each, the task being to write down the other word of each pair. For half of the Ss in each group. the cue words were the $\mathrm{R}$ words from VDL and. for the remaining half. they were the $W$ words. Five minutes were allowed for completion of the recall test. At the end of this time the $S$ was given a written questionnaire to complete on his own before leaving the laboratory. The questionnaire was modeled after that used by Rove and Paivio (1971b). whereby the Ss were asked to indicate. for each pair. which one of several possible strategies had been used to learn that pair during VDL. The strategy classifications. described in detail at the beginning of the questionnaire. Were repetition (covert rehearsal of the $R$ item). single imagery. compound imagery. single sentence. compound sentence, or "other."

\section{Verbal Discrimination Learning}

The VDL error scores were subjected to a 5 by 2 by 2 by 2 analysis of variance, with instructions. imagery level of pairs, trials, and cue type as factors. Cue type was a dummy factor. included as a check that the groups receiving $\mathrm{R}$ and $\mathrm{W}$ cues in associative recall were roughly equated on VDL performance. This was confirmed, since cue type was not significant as a main effect, nor did it interact with any other factor. The analysis showed that instruction was a significant source of variance $[F(4,50)=12.2]$. High-I pairs were easier than Low-I $[F(1.50)=53.8]$ and performance improved across trials $[F(1.50)=29.5$, all ps $<.001]$. None of the interactions involving these variables was significant. Collapsed across cue type and trials, the data are presented in Table 1. Multiple comparisons among the mean total errors for each instruction group were carried out using the Newman-Keuls procedure with an adopted significance level of .05 . The results are given in Table 1 . Briefly, SI and SS instructions facilitated performance relative to Conditions $\mathrm{CI}, \mathrm{CS}$, and the control, with no significant differences occurring within these two groupings. Thus, it appears that "single" instructions of the type used here benefit VDL, whereas "compound" instructions do not. These findings are, furthermore, independent of the I value of pairs.

As is apparent from Table 1. performance was essentially error free for High-I pairs learned under SI and SS instructions. Eight of the $12 \mathrm{Ss}$ in both conditions committed no errors on these pairs. Because of the possible influence of these two low-variance cells on the analysis of variance results, the data of Table 1 were reanalyzed following a square-root transformation to reduce heterogeneity of variance. The results were identical to those cited previously.

\section{Associative Recall}

The mean number of pairs recalled correctly on the associative recall test as a function of instructions, cue type, and I value of pairs is presented in Table 2. A three-factor analysis of variance of these data yielded significant main effects of instruction $[F(4.50)=15.5]$ and $I[F(1,50)=109]$ (both ps $<.001$ ) and two significant interactions: Instruction by Cue Type $[F(4.50)=2.68, \quad p<.05]$ and Instruction by $I$ $[F(4.50)=3.97, p<.01]$. An analysis of the square roots of the raw scores produced the same significant sources of variance. 
The nature of the Instruction by Cue Type interaction was examined by a series of post hoc comparisons among the means for the various conditions with the data collapsed across pair type. With the $\mathrm{R}$ word as the recall cue, performance was highest for Condition CS $(72 \%)$, followed in turn by the control (30\%), CI (27\%), SI $(19 \%)$, and SS $(8 \%)$. All of the between-group differences except for the control vs $\mathrm{CI}$ were significant by the Newman-Keuls test $(p<.05)$. For W-R recall, performance in the CS condition (50\%) again surpassed all other groups $(\mathrm{p}<.05)$. which did not differ among themselves $(\bar{X}=24 \%)$. The basis of the interaction can be seen most clearly from comparisons of R-cued vs W-cued recall within each instructional group. As indicated in Table 2, R-cued recall was better than W-cued recall in Condition CS $[\mathrm{t}(50)=3.93, \mathrm{p}<.001$, two-tailed], but the reverse was true for Condition SS $[\mathfrak{t}(50)=2.38, \quad \mathrm{p}<.05$, two-tailed $]$. Similar but nonsignificant trends were exhibited in Conditions CI and SI, respectively, thereby suggesting the general conclusion that cueing with the $\mathrm{R}$ item facilitates associative recall of pairs learned under "compound" instructions, whereas W-item cueing is superior with "single" instructions. These findings are in partial agreement with Rowe and Paivio (1971a), who reported significantly higher recall with $\mathrm{W}$-item cueing for single image instructions. However, recall under the compound image instruction used in that study was not significantly affected by the type of recall cue, although there was a slight superiority favoring W-cued items.

Separate pairwise comparisons across the five instruction groups for High-I and Low-I pairs produced parallel results in each case. i.e.. Condition CS was significantly better than the remaining conditions, which did not differ from each other. In addition. High-I words were recalled better than Low-I words in every condition, with the differences between the two pair types ranging from $10 \%$ in Condition SS to $30 \%$ in Condition CS. The variation in the size of these recall differences accounts for the significant Instruction by I interaction in the analysis of variance.

\section{Reported Strategies}

The mean number of pairs for which each type of strategy was reported as a function of instruction and pair type is shown in Table 3. These data are presented as an adjunct to the learning scores, since they exhibit some interesting and suggestive trends regarding strategy usage. Omissions. which accounted for $4.7 \%$ of the overall responses, and reports of "other" strategies. which accounted for $5.5 \%$ are not reported. The first point to be noted in Table 3 is that each of the experimental conditions produced the highest number of reports for the strategy that was primed by the instructional set. The pertinent figures are italicized in the table. This is encouraging but. of course. not definitive. since the influence of demand characteristics
Table 2

Mean Number of Words Correct in Associative Recall for Single Image (SI), Compound Image (CI), Single Sentence (SS), Compound Sentence (CS), and Control Conditions for Right (R-W) and Wrong (W-R) Cue Types (Experiment I)

\begin{tabular}{lcccrr}
\hline & \multicolumn{5}{c}{ Instruction } \\
\cline { 2 - 6 } Level & $\mathrm{SI}$ & $\mathrm{CI}$ & $\mathrm{SS}$ & $\mathrm{CS}$ & Control \\
\hline & \multicolumn{5}{c}{$\mathrm{R}-\mathrm{W}$} \\
High-I & 4.83 & 7.17 & 2.33 & 15.33 & 6.50 \\
Low-I & 1.17 & 1.33 & 0.33 & 7.67 & 3.17 \\
Total & 6.00 & 8.50 & 2.66 & 23.00 & 9.67 \\
& \multicolumn{7}{c}{$\mathrm{W}-\mathrm{R}$} \\
High-I & 7.00 & 6.33 & 4.17 & 10.33 & 5.00 \\
Low-I & 2.17 & 1.17 & 2.83 & 5.50 & 1.50 \\
Total & 9.17 & 7.50 & 7.00 & 15.83 & 6.50 \\
\hline
\end{tabular}

Note-Standard deviations ranged from .48 to 4.96

in determining reports of these strategies is unknown. The results for the control condition, however, and the distribution of choices among the nonprimed categories in the various instructional groups should be less affected by this type of artifact, so these data can be considered in more detail.

For the control group, the largest number of pairs were reportedly learned by either repetition or single imagery. This is true for both High-I and Low-I items. but there is a suggestion that repetition was favored for Low-I pairs and single imagery for High-I, as previous studies have shown (Rowe, 1971: Rowe \& Paivio, $1971 \mathrm{~b})$. An analysis of variance of reports of these two strategies alone. however, failed to produce a significant interaction in the present case $[\mathrm{F}(1,22)=3.41]$.

In the SI condition, reports of strategies other than single imagery were negligible for High-I pairs. For Low-I pairs, the instructional set was followed for about half the items, with deviations tending in the direction of repetition and single strategies about equally often. For the $\mathrm{CI}$ group, there was a marked tendency for Ss to substitute single imagery for the CI strategy with High-I pairs and to substitute single imagery and repetition about equally for Low-I pairs. In the SS condition. failures to use the instructed strategy are accounted for primarily by the use of single imagery for both pair types. while for the CS group the "displacement" strategies were predominantly compound imagery and single sentences for High-I pairs and repetition for Low-I pairs.

In sum, the subjective reports indicate a tendency to favor a single image strategy for High-I pairs and a repetition strategy for Low-I pairs in the absence of explicit mnemonic instructions. Additionally. deviations from instructional sets usually involved a switch to single imagery for High-! pairs and a switch to repetition for Low-I pairs. reinforcing the suggested dominance of these two types of strategies and their interaction with $\mathbf{I}$. The Ss appeared to follow SI instructions more completely than any other in the case of High-I pairs. while SS instructions were followed best for Low-I pairs. 
Table 3

Mean Percentage of Pairs for Which Repetition. Single Image (SI), Compound Image (CI). Single Sentence (SS), and Compound Sentence (CS) Strategies were Reported for Each Instruction Condition (Experiment I)

\begin{tabular}{ccrrrrr}
\hline \multirow{2}{*}{$\begin{array}{c}\text { Reported } \\
\text { Strategy }\end{array}$} & $\begin{array}{c}\text { Imagery } \\
\text { Level }\end{array}$ & SI & CI & SS & CS & $\begin{array}{c}\text { Con- } \\
\text { trol }\end{array}$ \\
\cline { 3 - 6 } Repetition & High-I & 1 & 6 & 4 & 9 & 22 \\
& Low-I & 21 & 26 & 8 & 21 & 32 \\
\multirow{3}{*}{ SI } & High-I & 91 & 34 & 44 & 5 & 36 \\
& Low-I & 48 & 29 & 15 & 11 & 29 \\
\multirow{2}{*}{ CI } & High-I & 1 & 35 & 9 & 19 & 13 \\
& Low-I & 1 & 13 & 9 & 3 & 7 \\
\multirow{2}{*}{ SS } & High-I & 4 & 8 & 39 & 18 & 5 \\
& Low-I & 25 & 14 & 68 & 14 & 5 \\
\multirow{2}{*}{ CS } & High-I & 0 & 1 & 2 & 57 & 9 \\
& Low-I & 1 & 6 & 1 & 48 & 7 \\
\hline
\end{tabular}

\section{Discussion}

The VDL results offer the general conclusion that instructions of the "single" variety used here, which encourage mnemonic processing of the $\mathrm{R}$ items alone. facilitate discrimination learning of both High-I and Low-I pairs, whereas compound instructions, which encourage processing of both items, do not. Consideration of the data in the light of the associative recall results and the subjective reports, however. suggests two important qualifications of this conclusion.

First. considering single instructions, the subjective reports (Table 3 ) indicate that the facilitation resulting from SI and SS instructions cannot be attributed solely to that strategy primed by the instructional set. The SI group tended to stick with the SI strategy for High-I pairs. but substituted the SS strategy for about $25 \%$ of the Low-I pairs. Conversely, the SS group in general used single sentences for Low-I pairs, but showed a pronounced tendency to switch to single imagery for High-I pairs. This "confounding" of these two strategies thus has a systematic form, in that the SI strategy seems to be preferred for discriminating High-I pairs and the SS strategy for Low-I pairs. The apparent equivalent effects of both types of single instructions on VDL performance for both types of pairs, therefore. may in fact reflect a differential use of imagery and sentence strategies for High-I and Low-I pairs, respectively. This pattern of strategy usage is consistent with Paivio's (1971) two-process theory, even though imagery vs sentence instructions had no differential effect on either VDL scores or associative recall as a function of I. As already pointed out, the latter interaction has been observed in intentional PAL (Paivio \& Foth, 1970) when special procedures are followed, i.e., forcing the Ss to use a particular strategy by requiring them to overtly produce their mediators. Earlier research on this problem failed to show the predicted interaction of Instruction by $I$. apparently because of Ss' tendencies to switch to more preferred strategies in the course of learning. in a way similar to that observed here (see Paivio. 1971. Chapter 11).

The second qualification of the instructions effect in VDL concems the CI condition. Contrary to the data reported by Rowe and Paivio (1971a). where CI instructions were also ineffective in VDL. this condition did not facilitate associative recall in the present experiment. If we view the associative recall data as a check on the use of compound imagery, the indication is that the Ss did not conform to the CI instructions. since this strategy clearly facilitates associative memory under other incidental learning conditions (e.g.. Yarmey \& Ure. 1971). Such an interpretation is corroborated by the questionnaire data, which show that the CI strategy was followed least well of all the instructional sets. Thus. the present experiment does not provide a clear test of the efficiency of a Cl strategy in VDL, except insofar as it illustrates an apparent unwillingness on the part of $S$ s to adopt this particular technique. An attempt was made in Experiment II to provide a stronger test of $\mathrm{CI}$ effectiveness in discrimination learning.

Finally. it should be noted that the CS instruction did facilitate associative recall and did seem to be used fairly consistently, as reflected in the subjective reports. It also produced no effect in the VDL phase of the experiment. although the CS group made fewer errors than the control, especially with High-I items. The possibility that CS instructions might facilitate both discrimination and association of pairs was also examined further in Experiment II.

\section{EXPERIMENT II}

The primary purpose of this experiment was to gain a greater degree of control over Ss' use of compound image and compound sentence strategies, so that their possible effects on VDL could be assessed more clearly than in Experiment I. The procedure used by Paivio and Foth (1970) was adopted, whereby the Ss were given $15 \mathrm{sec}$ to construct a compound image or compound sentence for each VDL pair and to either roughly draw their image or write down the sentence thus constructed. Two additional groups were instructed simply to form compound images or sentences without overt production. and a control group was given no mnemonic instructions. An associative recall test followed the VDL test trial, as in Experiment I.

\section{Method}

\section{Subjects}

Eighty paid volunteers were obtained from the same pool as used in Experiment I. The Ss. who were all naive regarding VDL experiments. Were assigned in rotation to one of the five experimental conditions: CI overt. CI covert. CS overt. CS covert. and control. with an equal number of males and females in each. 
Lists

Eighty words with I ratings greater than 6.03 and Thorndike-Lorge frequency values greater than 24 per million were chosen from the Paivio et al (1968) norms, and two separate 40-pair lists were constructed by the same method as in the first experiment.

\section{Procedure}

The Ss received one study-test trial on the VDL list. followed by an associative recall test. For VDL the pairs were typed on index cards and manually presented by $E$ for $15 \mathrm{sec}$ each, timed by a stopwatch. All Ss read both words aloud at the beginning of the study exposure. The CI-covert and CS-covert instructions were identical to the $\mathrm{CI}$ and $\mathrm{CS}$ instructions in Experiment I. i.e., Ss were told to form an image or sentence that incorporated both the R and W word of the pair but that in some way would help them remember the $\mathrm{R}$ word. In the $\mathrm{Cl}$-overt and $\mathrm{CS}$-overt conditions. they were instructed to draw their images and write their sentences in an answer booklet. one image (sentence) per page. Control Ss studied the list with no mention of possible mnemonic strategies. Practice on the VDL list using the appropriate strategies was provided by a study-test trial on a separate list of four High-I pairs. The procedure followed on the test trial was similar to Experiment I. With the exception that $10 \mathrm{sec}$ were allowed for responding instead of $6 \mathrm{sec}$. Half of the Ss in each group received one set of pairings and the remainder the other set. Also. half of the Ss receiving each list had one member of a pair correct and the other half had the other item correct. The spatial position of the items in half of the pairs was reversed on the test trial.

For the associative recall test. $E$ presented one word from each pair. typed on an index card. and the $S$ was allowed a maximum of $10 \mathrm{sec}$ to respond with the other word. Half of the Ss in each group received the $\mathrm{R}$ words from VDL as recall cues. while the remaining half received the $\mathrm{W}$ words.

\section{Results}

\section{Verbal Discrimination Learning}

The mean number of errors for each condition in VDL is shown in the upper portion of Table 4 . The analysis of variance showed that instruction produced a significant effect $[F(4.75)=3.73 . p<.01]$. Cue type in associative recall was also included as a dummy factor and produced no significant main effect or interaction. Newman-Keuls comparisons among the instruction means revealed a significant difference $(p<.05)$ only in the case of the CI-overt vs the CI-covert group. None of the instruction conditions differed reliably from the control.

\section{Associative Recall}

The mean number of pairs recalled correctly on the associative recall test was subjected to a 5 by 2 analysis of variance with instructions and cue type as factors. The only significant source of variance was the instruction variable. $[F(4.70)=23.2 . \quad p<.001]$. Newman-Keuls comparisons were performed on the instruction means with the data collapsed across cue type (Table 4). Associative recall for the Cl-overt and control conditions was equiralent. hut these were
Table 4

Mean Number of Errors in Verbal Discrimination Learning and Mean Number Correct in Associative Recall for the Compound Image (CI), Compound Sentence (CS), and Control Groups (Experiment II)

\begin{tabular}{cccccc}
$\begin{array}{c}\text { Type } \\
\text { of Test }\end{array}$ & $\begin{array}{c}\text { CI } \\
\text { Overt }\end{array}$ & $\begin{array}{c}\text { CI } \\
\text { Covert }\end{array}$ & $\begin{array}{c}\text { CS } \\
\text { Overt }\end{array}$ & $\begin{array}{c}\text { CS } \\
\text { Covert }\end{array}$ & Control \\
\hline VDL & $10.44^{\mathrm{a}}$ & $4.12^{\mathrm{b}}$ & $8.81^{\mathrm{a}, \mathrm{b}}$ & $6.31^{\mathrm{a}, \mathrm{b}}$ & $6.31^{\mathrm{a}, \mathrm{b}}$ \\
Recall & $7.00^{\mathrm{a}}$ & $18.88^{\mathrm{b}}$ & $27.94^{\mathrm{c}}$ & $30.00^{\mathrm{c}}$ & $10.38^{\mathrm{a}}$ \\
\hline
\end{tabular}

Note-a, $b$, and $c$ indicate means did not differ from others with same superscript for each type of test. The standard deviations ranged from 3.72 to 6.11 for VDL and from 5.87 to 10.75 for associative recall.

surpassed by the CI-covert group. Recall following CS-overt and CS-covert instructions was better than all other conditions, but these two did not differ significantly from each other.

\section{Discussion}

None of the instruction conditions affected VDL relative to the control group. Thus, requiring Ss to overtly produce their strategies did not enhance the effectiveness of these strategies; in fact, the CI-overt group had significantly more errors than the CI-covert group. However, the implications of this result are not completely clear because of the failure of CI-overt instructions to facilitate associative recall, as it should have had the Ss been forming compound images. The CI-overt group was significantly inferior to $\mathrm{CI}$ covert in associative recall. Thus, unlike its effect in intentional PAL (Paivio \& Foth. 1970), overt image production seems to interfere with association formation when superimposed upon VDI. This outcome is not attributable to a reluctance on the part of Ss to draw their images: An examination of the drawings showed that images were produced for about $88 \%$ of the pairs. Apparently, requiring overt production of compound images during VDL did not result in a greater use of such mediators by the Ss. This attempt to force the use of compound imagery in VDL must, therefore, be classed as a failure. Note, however, that the Cl-covert instructions. which facilitated associative recall. also produced no effect in discrimination learning. This finding replicates the results of Rowe and Paivio (1971a) and indicates that compound imagery, whether or not accompanied by specific tagging instructions, does not benefit VDL.

The data obtained for the two CS groups are somewhat more definitive than the $\mathrm{CI}$ results. Both CS-overt and CS-covert conditions were equivalent in their effects on both VDL and associative recall. The average level of performance on the recall test for the two sentence conditions was about $73 \%$, almost three times as high as the control group. suggesting that the $\mathrm{Ss}$ were using the CS strategy as instructed. The CS-overt group in fact wrote complete sentences for about 72 of the pairs. The null effect of CS instructions in VDL. 
coupled with their large effect in associative recall both here and in Experiment $I$. is a strong indication that this type of strategy does not facilitate discrimination learning.

\section{GENERAL DISCUSSION}

Three main conclusions arise from the study. First. instructions that direct the $S$ to use either images or sentences in elaborative processing of the $R$ items in VDL improve performance relative to an uninstructed control condition. Experiment I showed that this effect was independent of the I value of the word pairs. but reports of Ss' learning strategies suggested a differential usage of imagery and sentences for High-I and Low-I pairs in a way consistent with the theory that High-I items are more effectively processed via imagery, while Low-I items benefit more from verbal mediation (Paivio, 1971).

Second. the results of both experiments are consistent regarding the effects of a compound sentence strategy in VDL. Incorporating both words of each pair into a simple sentence enhanced associative recall but did not affect discrimination learning. These results compare favorably with previous evidence showing the superiority of sentence mediators in PAL (e.g.. Rohwer \& Levin, 1968 ) but indicate that attempts to produce facilitation of both discrimination and associative learning of pairs concurrently by this technique may prove futile.

Third, the weight of the evidence also points to the ineffectiveness of compound imagery in VDL, although this conclusion is weakened somewhat by the difficulty in inducing a reliable use of the CI strategy. This was apparent in Experiment I and in the CI-overt condition in Experiment II. where the instructions affected neither VDL nor associative recall. This point has already been discussed: suffice it to say here that, in two instances where $\mathrm{Cl}$ instructions did facilitate associative recall (the CI-covert condition in Experiment II; Rowe \& Paivio, 1971a). a null effect in VDL still obtained. The overall conclusion. then. is that compound imagery is an ineffective strategy in discrimination learning. It should be mentioned that. in a subsequent unpublished experiment. we have obtained significantly higher performance with CI instructions in VDL, but the effect was accompanied by a failure of the instructions to enhance associative recall. These data are, therefore, indeterminant, since again the indication is that the $\mathrm{CI}$ instructions did not successfully activate the use of a $\mathrm{Cl}$ strategy.

The effects of SI and SS instructions might be accounted for by the frequency theory of VDL (Ekstrand, Wallace, \& Underwood, 1966), if it is assumed that constructing an image or sentence for a word increases its subjective frequency. There are, however, difficulties with such an interpretation. Consider first the SI effect. According to frequency theory, any situation that gives rise to a higher situational frequency for the $\mathrm{R}$ item of a pair will enhance VDL. Whether image formation increases item frequency compared to nonimaged items in the same list is not known. but it does not when frequency judgments of imaged and nonimaged words are compared between different groups of Ss (Ghatala, Levin. \& Wilder. 1973). Furthermore, even if imagery is shown to affect frequency estimation when manipulated within Ss. this would not require an interpretation of the SI effect in terms of frequency theory. since other research has indicated that the effects of imagery and frequency in VDL are independent. i.e. performance differences associated with either of these variables cannot be easily explained in terms of the other (Rowe, 1972).

The effect of SS instructions lends itself more readily to a frequency theoretic interpretation. since producing a sentence containing the $R$ word should increment the subjective frequency of the word by one. In Experiment I, where Ss pronounced both intrapair items aloud once during the study presentation, sentence formation would, therefore, result in a $2: 1$ ratio of responses favoring the $\mathrm{R}$ items as compared to a $1: 1$ ratio for control pairs. This frequency differential might be sufficient to explain the superior performance exhibited by the SS group. but we doubt that it is. since frequency differences of one between the $\mathrm{R}$ and $\mathrm{W}$ words do not facilitate VDI (Cole \& Kanak. 1972). Furthermore, this analysis of the distribution of frequency units does not include rehearsal of the correct alternative responses, which may or may not occur in the SS condition but which, if they occur in the control condition, as would be predicted by frequency theory. will give a 2:1 ratio for control pairs as well.

Thus, it is argued that frequency theory does not give a satisfactory account of the effects of SI and SS instructions in VDL. An alternative interpretation may be found in the "feature tagging" or differential encoding class of explanations (Kausler. 1973; Rowe, 1972), which postulate that Ss learn a verbal discrimination by attending to the nonoverlapping attributes or features of the $\mathrm{R}$ and $\mathrm{W}$ items within each pair. In the present case. both image and sentence formation should produce distinctive semantic features for the $\mathrm{R}$ item, which can serve to differentiate it from the $W$ item and, hence, to aid the discrimination process. The differential encoding hypothesis has not been developed to the same extent or with the same rigor as frequency theory, nor has it been subjected to the same degree of empirical test, but it does seem capable of explaining some of the findings not readily accounted for in terms of subjective frequency differences and, hence, deserves further experimental attention.

\section{REFERENCES}

Cole. L. E.. \& Kanak, N. J. Overticovert pronunciation and rehearsal in verbal-discrimination learning. American Journal of Psychology. 1972. 85. 57-61 
Lkitrand. B. R. Wallace. W. P.. \& Lnderwood. B. J. A frequency theory of verbal discrimination learning. Psychological Reriew. 1966.73.566-578.

Ghatala. E. S., Levin, J. R., \& Wilder, L. Apparent frequency of words and pictures as a function of pronunciation and imagery. Journal of Verbal Learning \& Verbal Behavior. 1973. 12. $85-90$

Kausler. D. H. Homographs as double function items in verbal discrimination learning. Journal of Experimental Psychologs: 1973. 98. 214-216

Levin. J. R., Ghatala. E. S.. Wilder. L.. \& Inzer. E. Imagery and vocalization strategies in children's verbal discrimination learning. Journal of Educational Psychology. 1973. 64. $360-365$

Paivio. A. Imagery and rerbal processes. New York: Holt, Rinehart.\& Winston. 1971

Paivio. A., \& Foth. D. Imaginal and rerbal mediators and noun concreteness in paired-associate learning: The elusive interaction. Journal of Verbal Learning \& Verbal Behavior. 1970. 9. 384-390.

Paivio. A.. Yuille. J. C.. \& Madigan. S. Concreteness, imagery. and meaningfulness values for 925 nouns. Journal of Experimental Psychology Yonograph Supplement. 1968. 76(1. Part 2).

Rohwer. W. D.. \& Levin. J. R. Action. meaning. and stimulus selection in paired-associate learning. Journal of Verbal
Learning \& Verbal Behavior. 1968. 7. 137-141.

Rowe. E. J. Verbal discrimination learning as a function of noun imagery and an induced frequency strategy. Paper presented at the meeting of the Canadian Pstchological Association, St. John's. June 1971.

Rowe. I. J. Imagery and frequency processes in verbal discrimination learning. Journal of Experimental Psychology, 1972, 95. 140-146.

Rowe. E. J.. \& Paivio, A. Imagery and repetition instructions in verbal discrimination and incidental paired-associate learning. Journal of Verbal Learning \& Verbal Behavior, 1971a. 10, 668-672.

Rowe. E. J.. \& Paivio, A. Word frequency and imagery effects in verbal discrimination learning. Journal of Experimental Psichology, 1971b. 88, 319-326.

Wike. S. S., \& Wike, E. L. The effect of a coding cue on verbal discrimination learning. Psi'chonomic Science. 1970. 20. 107-108.

Yarmey, A. D. \& L ire. G. Incidental learning, noun imagery-concreteness and direction of associations in paired-associate learning. Canadian Journal of Psychology. 1971.25.91-102.

(Received for publication July 25. 1973; accepted July 29.1973.) 